\title{
DYNAMIC WORMHOLE SPACETIMES COUPLED TO NONLINEAR ELECTRODYNAMICS
}

\author{
AARÓN V. B. ARELLANO \\ Facultad de Ciencias, Universidad Autónoma del Estado de México, \\ El Cerrillo, Piedras Blancas, C.P. 50200, Toluca, México \\ vynzds@yahoo.com.mx \\ FRANCISCO S. N. LOBO \\ Centro de Astronomia e Astrofísica da Universidade de Lisboa, \\ Campo Grande, Ed. C8 1749-016 Lisboa, Portugal \\ flobo@cosmo.fis.fc.ul.pt
}

\begin{abstract}
We explore the possibility of dynamic wormhole geometries, within the context of nonlinear electrodynamics. The Einstein field equation imposes a contracting wormhole solution and the obedience of the weak energy condition. Furthermore, in the presence of an electric field, the latter presents a singularity at the throat, however, for a pure magnetic field the solution is regular. Thus, taking into account the principle of finiteness, that a satisfactory theory should avoid physical quantities becoming infinite, one may rule out evolving wormhole solutions, in the presence of an electric field, coupled to nonlinear electrodynamics.
\end{abstract}

Keywords: Traversable wormholes; nonlinear electrodynamics.

Pioneering work on nonlinear electrodynamic theories may be traced back to Born and Infeld, ${ }^{1}$ where the latter outlined a model to remedy the fact that the standard picture of a point charged particle possesses an infinite self-energy. Therefore, the Born-Infeld model was founded on a principle of finiteness, that a satisfactory theory should avoid physical quantities becoming infinite. Recently, nonlinear electrodynamics has found a wide range of applicability, namely, as effective theories at different levels of string/M-theory, cosmological models, black holes, and in wormhole physics, amongst others ( $\mathrm{see}^{2,3}$ and references therein).

Relatively to wormhole physics it was found that static spherically symmetric and stationary axisymmetric traversable wormholes cannot exist within nonlinear electrodynamic, mainly due to the presence of event horizons, the non-violation of the null energy condition at the throat, and due to the imposition of the principle of finiteness. ${ }^{3,4}$ In this work, we shall explore the possibility that nonlinear electrodynamics may support time-dependent traversable wormhole geometries. This is of particular interest as the energy conditions are not necessarily violated for evolving 
wormhole spacetimes. ${ }^{5}$

The action of $(3+1)$-dimensional general relativity coupled to nonlinear electrodynamics is given by (with $G=c=1$ )

$$
S=\int \sqrt{-g}\left[\frac{R}{16 \pi}+L(F)\right] d^{4} x
$$

where $R$ is the Ricci scalar. $L(F)$ is a gauge-invariant electromagnetic Lagrangian, depending on a single invariant $F$ given by $F=F^{\mu \nu} F_{\mu \nu} / 4$, where $F_{\mu \nu}$ is the electromagnetic tensor. Note that in Einstein-Maxwell theory, the Lagrangian takes the form $L(F)=-F / 4 \pi$.

Varying the action with respect to the gravitational field provides the Einstein field equations $G_{\mu \nu}=8 \pi T_{\mu \nu}$, with the stress-energy tensor given by

$$
T_{\mu \nu}=g_{\mu \nu} L(F)-F_{\mu \alpha} F_{\nu}{ }^{\alpha} L_{F},
$$

where $L_{F}=d L / d F$.

We shall consider that the spacetime metric representing a dynamic spherically symmetric $(3+1)$-dimensional wormhole, which is conformally related to the static wormhole geometry, ${ }^{6}$ takes the form

$$
d s^{2}=\Omega^{2}(t)\left[-e^{2 \Phi(r)} d t^{2}+\frac{d r^{2}}{1-b(r) / r}+r^{2}\left(d \theta^{2}+\sin ^{2} \theta d \phi^{2}\right)\right]
$$

where $\Phi$ and $b$ are functions of $r$, and $\Omega=\Omega(t)$ is the conformal factor, which is finite and positive definite throughout the domain of $t$. To be a wormhole solution, the following conditions are imposed: $\Phi(r)$ is finite everywhere in order to avoid the presence of event horizons; $b(r) / r<1$, with $b\left(r_{0}\right)=r_{0}$ at the throat; and the flaring out condition $\left(b-b^{\prime} r\right) / b^{2} \geq 0$, with $b^{\prime}\left(r_{0}\right)<1$ at the throat.

For this particular case, the weak energy condition, which is defined as $T_{\mu \nu} U^{\mu} U^{\nu} \geq 0$ where $U^{\mu}$ is a timelike vector, is satisfied, ${ }^{2}$ contrary to the static and spherically symmetric traversable wormholes. ${ }^{3,4}$

Through the Einstein field equation, we obtain the following relationship

$$
\frac{b^{\prime} r-b}{2 r^{3}}=-\left[2(\dot{\Omega} / \Omega)^{2}-\ddot{\Omega} / \Omega\right],
$$

which provides the solutions

$$
b(r)=r\left[1-\alpha^{2}\left(r^{2}-r_{0}^{2}\right)\right], \quad \Omega(t)=\frac{2 \alpha}{C_{1} e^{\alpha t}-C_{2} e^{-\alpha t}},
$$

where $\alpha$ is a constant, and $C_{1}$ and $C_{2}$ are constants of integration. Now, $\Omega(t) \rightarrow 0$ as $t \rightarrow \infty$, which reflects a contracting wormhole solution. This analysis shows that one may, in principle, obtain an evolving wormhole solution in the range of the time coordinate. A fundamental condition to be a solution of a wormhole, is that $b(r)>0$ is imposed. ${ }^{7}$ Thus, the range of $r$ is $r_{0}<r<a=r_{0} \sqrt{1+1 / \beta^{2}}$, with $\beta=\alpha r_{0}$. If $a \gg r_{0}$, i.e., $\beta \simeq r_{0} / a \ll 1$, one may have an arbitrarily large wormhole. Note, however, that one may, in principle, match this solution to an exterior vacuum solution at a junction interface $R$, within the range $r_{0}<r<a$. 
The electromagnetic field equations take the following form

$$
\left(F^{\mu \nu} L_{F}\right)_{; \mu}=0, \quad\left({ }^{*} F^{\mu \nu}\right)_{; \mu}=0 .
$$

Taking into account the symmetries of the geometry, the non-zero compatible terms for the electromagnetic tensor are $F_{\mu \nu}=2 E\left(x^{\alpha}\right) \delta_{\mu}^{[t} \delta_{\nu}^{r]}+2 B\left(x^{\alpha}\right) \delta_{\mu}^{[\theta} \delta_{\nu}^{\phi]}$, where $F_{t r}=$ $E$ is the electric field, and $F_{\theta \phi}=B$, the magnetic field. From the electromagnetic field equations, we deduce the following

$$
E(t, r)=\frac{f \Omega^{2} r \pm \sqrt{f^{2} \Omega^{4} r^{2}-\left(32 \pi q_{\mathrm{e}} q_{\mathrm{m}}\right)^{2}}}{32 \pi q_{\mathrm{e}} r^{2}(1-b / r)^{1 / 2}}, \quad B(\theta)=q_{\mathrm{m}} \sin \theta,
$$

with $f=\left(b^{\prime} r-3 b\right)$, and $q_{\mathrm{e}}$ and $q_{\mathrm{m}}$ are constants related to the electric and magnetic charge, respectively. From this solution we point out two observations: (i) the requirement of $f^{2} \Omega^{2} r^{2}>\left(32 \pi q_{\mathrm{e}} q_{\mathrm{m}}\right)^{2}$; (ii) and $E \propto(1-b / r)^{-1 / 2}$, showing that the $E$ field is singular at the throat, which is in contrast to the principle of finiteness.

An interesting case arises considering a pure magnetic field, $E=0$, from which we obtain the Lagrangian and its derivative

$$
L=-\frac{1}{8 \pi \Omega^{2}}\left[b^{\prime} / r^{2}+3(\dot{\Omega} / \Omega)^{2}\right], \quad L_{F}=\frac{1}{16 \pi q_{\mathrm{m}}^{2}} \Omega^{2} r\left(b^{\prime} r-3 b\right) .
$$

These equations, together with $B=q_{\mathrm{m}} \sin \theta, F=q_{\mathrm{m}}^{2} /\left(2 \Omega^{4} r^{4}\right)$ and solutions (5) provide a regular wormhole solution at the throat, with finite fields. We emphasize that this result is in close relationship to the regular magnetic black holes coupled to nonlinear electrodynamic found by Bronnikov. ${ }^{4}$

In conclusion, we have explored the possibility of evolving time-dependent wormhole geometries coupled to nonlinear electrodynamics. It was found that the Einstein field equation imposes a contracting wormhole solution and that the weak energy condition is satisfied. In the presence of an electric field, a problematic issue was verified, namely, that the latter becomes singular at the throat. However, regular solutions of traversable wormholes in the presence of a pure magnetic field were found. Another point worth noting is that we have only considered that the gauge-invariant electromagnetic Lagrangian $L(F)$ be dependent on a single invariant $F$. It would also be worthwhile to include another electromagnetic field invariant $G \sim{ }^{*} F^{\mu \nu} F_{\mu \nu}$, which would possibly add an interesting analysis to the solutions found in this work.

\section{References}

1. M. Born, Proc. Roy. Soc. Lond. A143, 410 (1934); A144, 425 (1934); M. Born and

L. Infeld, Proc. Roy. Soc. Lond. A147, 522 (1934)

2. A. V. B. Arellano and F. S. N. Lobo, Class. Quant. Grav. 23 5811-5824 (2006).

3. A. V. B. Arellano and F. S. N. Lobo, Class. Quant. Grav. 23 7229-7244 (2006).

4. K. A. Bronnikov, Phys. Rev. D 63, 044005 (2001).

5. S. Kar, Phys. Rev. D 49, 862 (1994).

6. M. Morris and K.S. Thorne, Am. J. Phys. 56, 395 (1988).

7. J. P. S. Lemos, F. S. N. Lobo and S. Q. de Oliveira, Phys. Rev. D 68, 064004 (2003). 